\title{
MORBIDITY PATTERNS AND OUTCOME IN CHILDREN WITH SEVERE ACUTE MALNUTRITION AT A TERTIARY CARE HOSPITAL.
}

\footnotetext{
1. MBBS, FCPS (Pediatrics Medicine) Assistant Professor of Pediatrics Medicine Ghurki Trust Teaching Hospital, Lahore.

2. MBBS, FCPS (Pediatrics Medicine) Assistant Professor Pediatrics Unit-I Children Hospital Chandka Medical College/

SMBBMU, Larkana

3. FCPS (Pediatrics Medicine) Fellow in Pediatrics Neurology Agha Khan Hospital, Karachi.

4. MBBS, FCPS (Pediatrics Medicine) PGR Pediatric Medicine

The Children's Hospital and

The Institute of Child Health, Multan.
}

Correspondence Address:

Dr. Fazal Ur Rehman

Department of Pediatric Medicine

The Children's Hospital and

The Institute of Child Health, Multan.

fazal171@gmail.com

Article received on:

08/04/2019

Accepted for publication:

10/10/2019

\section{Waqas Ali ${ }^{1}$, Asif Ali Khuhro², Sanam Bano Rajper ${ }^{3}$, Fazal Ur Rehman ${ }^{4}$}

ABSTRACT... Objectives: We planned this study to find out morbidity patterns as well as outcome of admitted children aged less than 5 years of age with severe acute malnutrition (SAM). Study Design: Cross Sectional study. Setting: Department of Pediatrics Medicine, Ghurki Trust Teaching Hospital, Lahore. Period: From $1^{\text {st }}$ January 2018 to $30^{\text {th }}$ June 2018. Material \& Methods: A total of 80 children aged 1 to 59 months, of both genders, having SAM were considered. Physical examination, detailed clinical as well as laboratory findings related to nutritional status and comorbidities were noted. Outcome in terms of recovery/discharge, left against medical advice (LAMA) or death during the stay was noted in all the children. Results: Out of a total of 80 children, $20(25.0 \%)$ were aged 1 to 6 months, $39(48.8 \%)$ from 7 to 24 months and $21(26.2 \%)$ from 25 to 59 months. Most of the patients, $39(48.8 \%)$ were from 7 to 24 months of age, $62(77.5 \%)$ were admitted through emergency, 52 (65.0\%) had WHZ score4SD and 27 (33.8\%) using bottle feeding. Severe wasting without having edema was reported in $67(83.8 \%)$ children. Diarrhea was the commonest comorbidity, found in $43(53.8 \%)$ children, followed by sepsis 17 (21.3\%) and pneumonia 15 (18.8\%). There were 69 (86.3\%) children noted having anemia. Hyponatremia was the commonest metabolic abnormality seen in 40 $(25.0 \%)$ children. In terms of outcome, $71(88.7 \%)$ children recovered well and got discharged while mortality was reported in $5(6.3 \%)$ children. Conclusion: Diarrhea along with sepsis and pneumonia along with metabolic abnormalities were found to be more prevalent amongst children having SAM. Early identification of anemia as well as infections in children with SAM can contribute to better outcome.

Key words: $\quad$ Anemia, Diarrhea, Pneumonia, Severe Acute Malnutrition, Sepsis.

Article Citation: Ali W, Khuhro AA, Rajper SB, Fazal Ur Rehman. Morbidity patterns and outcome in children with severe acute malnutrition at a Tertiary Care Hospital. Professional Med J 2020; 27(9):1799-1803.

DOI: $10.29309 / \mathrm{TPMJ} / 2020.27 .09 .3528$

\section{INTRODUCTION}

Child malnutrition is a global problem and posing serious problems to children especially under the age of 5 years. In Asia, wasting is noted to be prevalent around $4 \%$ in children having age of less than 5 years while in low or middle income countries, this prevalence is around $3 \% .^{1,2}$

It is estimated that almost half of the deaths in children aged less than 5 years are due to the underlying malnutrition as more than 3.5 million deaths are being reported annually from around the world due to malnutrition. ${ }^{2-4}$ Not only it is major factor contributing to child mortality but it also damages cognitive as well as physical development, leave alone the economic side of it contributing to the whole society. ${ }^{5}$ It has been documented that around $80 \%$ of children who have malnutrition hail from countries which are lower or middle income ones. Severe Acute malnutrition (SAM) indicates an emergency condition that needs earliest attention while United Nations has estimated acute malnutrition to affect around $8 \%$ of children across the globe. ${ }^{6}$

In a country like Pakistan, malnutrition in children is attributed factors which are of socio-economic, environmental as well as political nature. Ithas been observed regularly in local studies that significant ratio of children admitting in healthcare facilities are found to have SAM while presenting illness may go on to develop nutritional deterioration 
which may result in poor outcomes. ${ }^{7}$ Rise in need of identifying morbidity patterns early in such neonates are bound to provide us ways for early diagnosis and management of children affected with SAM so this study was planned to find out morbidity patterns and outcome of admitted children aged less than 5 years with SAM.

\section{MATERIAL \& METHODS}

This was a cross sectional study conducted at the Department of Pediatrics Medicine, Ghurki Trust Teaching Hospital, Lahore from $1^{\text {st }}$ January 2018 to $30^{\text {th }}$ June 2018. Approval from the ethical committee of the institution was taken for this study. Consent from parents/guardians of all stu0dy participants was also acquired.

A total of 80 children aged 1 to 59 months, of both genders, having SAM described in terms of either weight for height $\mathrm{Z}$ score (WHZ) less than -3 with or without bilateral pitting edema and any of the these: anorexia, severe anemia, high grade fever, severely dehydrated and with systemic infection. ${ }^{7}$ Children with congenital malformation, neurological impairment or any medical as well as surgical illness obstructing oral / nasograstric feeding were not included in this study.

Physical examination was done while detailed clinical as well as laboratory findings related to nutritional status and comorbidities were also done. Demographics like age, gender, $\mathrm{WHZ}$ score, weight, height, duration of hospital stay and presenting complaints of all the participants were noted. Evaluation for identification of any mobidity as well as associated congenital or hereditary disorders was done on daily basis during the hospital stay of all the children.

WHO standards were applied for the management of SAM in all children. ${ }^{8}$ Nasograstric tube feeding was adopted for children who could not feed orally or appeared too sick. Vitamin A orally as 200,000 IU for children who were more than 12 months of age or 100,000 IU for children who were less than 12 months of age was administered at the time of admission to all the children who were presented with signs of deficiency of Vitamin A whereas further doses of same strength were repeated on day 2 and 14. Dehydration was managed with Rehydration Solution for Severe malnutrition (RESOMAL). First phase of management (3 to 4 days) involved therapeutic milk F-75 while continuation had F-100 milk or ready to use therapeutic food (RUTF) in the second phase based on the appetite. For infants who were less than 6 months of age, breast feeding, infant formula milk as well as diluted F-100 was utilized. Investigations like complete blood count along with blood glucose, complete urinalysis as well as electrolytes were ordered for all the participants. Further investigations like chest $x$-ray, blood culture, urine culture along with sensitivity, arterial blood gases or ultrasonography of the abdomen was ordered if needed depending upon the clinical situation of the children.

Penicillin along with gentamicin or ceftriaaxone and amikacin were used as antibiotic in almost all the participants of this study. Outcome in terms of recovery/discharge, left against medical advice (LAMA) or death during the stay was noted in all the children.

SPSS version 21 was used for data handling and analysis. Quantitative variable were represented in terms of means and standard deviation while qualitative variables were presented as frequency and percentages.

\section{RESULTS}

Out of a total of 80 children, 20 (25.0\%) were aged 1 to 6 months, 39 (48.8\%) from 7 to 24 months and $21(26.2 \%)$ from 25 to 59 months. Most of the patients, $62(77.5 \%)$ were admitted through emergency, 52 (65.0\%) had WHZ score-4SD and 27 (33.8\%) using bottle feeding. Severe wasting without having edema was reported in 67 (83.8\%) children while 13 (16.2\%) were having edematous malnutrition. All the baseline characteristics are detailed in Table-l.

There 48 (60.0\%) children who were febrile (100 to $104^{\circ} \mathrm{F}$ ) at the time of admission. As far as major morbidities amongst children with SAM were concerned, diarrhea was the commonest as found in $43(53.8 \%)$ children, followed by sepsis 17 (21.3\%) and pneumonia 15 (18.8\%). 
Eight $(10.0 \%)$ children with SAM had measles along with diarrhea and pneumonia. Tuberculosis was diagnosed in two cases while one case was having TB meningitis. Rickets confirmed by biochemical as well as radiological changes was found in $4(5.0 \%)$ cases.

There were $42(52.5 \%)$ children with SAM who were noted to have severe dehydration whereas remaining had some or no dehydration. In 17 cases with Sepsis, most (14) were having leucocytosis. Blood culture was seen 3 patients where klebsiella pneumonia was found in 2 and streptococcus pyogens in 1 case.

There were 69 (86.3\%) children noted having anemia, amongst which, $6(7.5 \%)$ were having hemoglobin less than $4 \mathrm{gm} / \mathrm{dl}, 21$ (30.4\%) between 4.4 to $7 \mathrm{gm} / \mathrm{dl}$ and $42(60.9 \%)$ with 7.1 to $10 \mathrm{gm} / \mathrm{dl}$.
Hyponatremia was the commonest metabolic abnormality seen in $40(25.0 \%)$ children with SAM while hypokalemia was reported in 13 (16.2\%) and hypoglycemia in 7 (8.8\%).

We noted 7 (8.8\%) children to have central nervous system disorder like hydrocephalus $(n=1)$, porencephalic cyst $(n=1)$ and seizure disorders $(n=5)$. Three children were noted having congenital heart disease, out of which, 1 had ventricular septal defect while 2 were having pulmonary hypertension. Nephrocalcinosis $(n=1)$ and urolithiasis $(n=1)$ were found in 2 patients.

Mean duration of hospital stay was recorded as 8.3 days with standard deviation of 3.87 days. In terms of outcome, 71 (88.7\%) children recovered well and got discharged (advised outpatient follow up) while $4(5.0 \%)$ LAMA. Mortality was reported in $5(6.3 \%)$ children, mainly due to diarrhea $(n=2)$, sepsis $(n=2)$ and measles pneumonia $(n=1)$.

\begin{tabular}{|c|c|c|c|c|}
\hline \multirow{2}{*}{ Characteristics } & \multicolumn{3}{|c|}{ Age (Months) } & \multirow{2}{*}{ Total $(n=80)$} \\
\hline & $1-6(n=20)$ & $7-24(n=39)$ & $25-59(n=21)$ & \\
\hline \multicolumn{5}{|c|}{ Gender } \\
\hline Male & $12(60.0 \%)$ & $26(66.7 \%)$ & $12(57.1 \%)$ & $50(62.5 \%)$ \\
\hline Female & $8(40.0 \%)$ & $13(33.3 \%)$ & $9(42.9 \%)$ & $30(37.5 \%)$ \\
\hline \multicolumn{5}{|l|}{ Mode of Admission } \\
\hline Outpatient & $1(5.0 \%)$ & $10(25.6 \%)$ & 7 (33.3\%) & $18(22.5 \%)$ \\
\hline Emergency & $19(95.0 \%)$ & $29(74.4 \%)$ & $14(66.7 \%)$ & $62(77.5 \%)$ \\
\hline \multicolumn{5}{|l|}{ WHZ Score } \\
\hline $3 \mathrm{SD}$ & 8 & 12 & 8 & $28(35.0 \%)$ \\
\hline $4 \mathrm{SD}$ & 12 & 27 & 13 & $52(65.0 \%)$ \\
\hline \multicolumn{5}{|l|}{ Edema } \\
\hline Yes & 2 & 7 & 4 & $13(16.2 \%)$ \\
\hline No & 18 & 32 & 17 & $67(83.8 \%)$ \\
\hline \multicolumn{5}{|c|}{ Feeding At the Time of Admission } \\
\hline Breast Feeding & 10 & 7 & 0 & $17(21.2 \%)$ \\
\hline Bottle Feeding & 10 & 13 & 4 & $27(33.8 \%)$ \\
\hline Semi-Solids Only & 0 & 5 & 13 & $18(22.5 \%)$ \\
\hline Breast Feeding \& Semi Solids & 1 & 14 & 3 & $18(22.5 \%)$ \\
\hline Mean Height $(\mathrm{cm})$ & 53.7 & 64.9 & 78.5 & 65.4 \\
\hline Mean Weight (kg) & 3.8 & 5.2 & 6.4 & 4.9 \\
\hline \multicolumn{5}{|c|}{ Table-I. Baseline Characteristics amongst Children with SAM. } \\
\hline \multicolumn{3}{|c|}{ Morbidity } & \multicolumn{2}{|c|}{ Number (\%) } \\
\hline \multicolumn{3}{|l|}{ Diarrhea } & \multicolumn{2}{|c|}{$43(53.8)$} \\
\hline \multicolumn{3}{|l|}{ Sepsis } & \multicolumn{2}{|c|}{$17(21.3 \%)$} \\
\hline \multicolumn{3}{|l|}{ Pneumonia } & \multicolumn{2}{|c|}{$15(18.8 \%)$} \\
\hline \multicolumn{3}{|l|}{ Measles } & \multicolumn{2}{|c|}{$8(10.0 \%)$} \\
\hline \multicolumn{2}{|l|}{ Rickets } & & \multicolumn{2}{|c|}{$4(5.0 \%)$} \\
\hline
\end{tabular}




\section{DISCUSSION}

In the present study, we noted, children having severe wasting as $83.8 \%$ while $16.2 \%$ were having edematous malnutrition. These figures correlates well with other studies conducted in Asia where most have recorded severe wasting around $75 \%$ cases while edematous malnutrition ranges around $25 \% .^{9,10}$ Some studies from African countries noted an even higher proportion of edematous malnutrition ranging from 50 to $70 \% .{ }^{11,12}$

SAM has been found to be more prevalent among boys in comparison to girls around the world. ${ }^{7,11,12}$ The reason is unknown for this male predominance but some studies from Pakistan also reported higher proportion of females with SAM as well. ${ }^{13,14}$

We noted 39 (48.8\%) children in age range of 7 to 24 months, children of this age range are more prone have SAM as has been depicted in various other findings. ${ }^{11,12}$ It has also been recorded that children of age 6 to 24 months are more likely to be presenting with malnutrition and a combo of likely co-infection. ${ }^{7}$ Fever is not always accompanied with SAM as study conducted from Zambia showed that $48 \%$ of children were febrile. We noted $48(60.0 \%)$ of the children having fever at the time of admission. ${ }^{12,15}$

Our results were consistent in relation to many other studies where diarrhea turned out to be the commonest morbidity accompanying children with SAM. ${ }^{7,12,16}$ Diarrhea is considered to be the commonest cause of mortality in children having SAM as well. This was again proved in the present study as 2 out of 5 deaths were attributed to diarrhea.

Ejaz and coworkers ${ }^{10}$ as well as Hossain and Colleagues $^{9}$ found Pneumonia to be another major morbidity found in children with SAM. In the present study, $18.8 \%$ were having pneumonia. Breast feeding is not always practiced in our area as was found that only $(21.2 \%)$ children were breastfeeding at the time of admission, which could be a reason contributing to low immunity as well as overall physical and neurodevelopment growth of the child. Lack of proper immunization and bad hygienic as well as sanitary practices also contribute to infections in these children.

Sepsis was found to be another major comorbidity in the present study as $21.3 \%$ children were noted with it. Figures around $15 \%$ are documented in children having sepsis with SAM. ${ }^{7,9}$ Leucocytosis is reported in around $80 \%$ of children with sepsis in SAM by other researchers from Pakistan ${ }^{7,10}$ as was found to be the case in the present study.

As far as limitations of the present study are concerned, comparatively small sample size could be a reason that studies with bigger sample size as well as conducted at multiple centers will further verify our findings. We did not have any controls to compare our cases that could potentially indicate some risk factors of SAM in the studied population as well.

Education and awareness related to overall hygienic practices, breastfeeding and adequate immunization should be the way forward for population of our area. Community programs, especially involving women should also be organized where the important risk factors as well as the preventive measures to defend against malnutrition amongst pediatric population can provide good basis to reduce morbidity as well as mortality related to malnutrition.

\section{CONCLUSION}

Diarrhea along with sepsis and pneumonia along with metabolic abnormalities were found to be more prevalent amongst children having SAM. Early identification of anemia as well as infections in children with SAM can contribute to better outcome.

\section{Copyright $(10$ Oct, 2019.}

\section{REFERENCES}

1. Pravana NK, Piryani S, Chaurasiya SP, Kawan R, Thapa RK, Shrestha S. Determinants of severe acute malnutrition among children under 5 years of age in Nepal: A communitybased case-control study. BMJ Open 2017; 7:e017084. 
2. Ministry of Health and Population. Nepal health sector program-implementation plan II (NHSP-IP2) 20102015: Ministry of health and population. Government of Nepal, 2010; 312010.

3. Horton R, Lo S. Nutrition: a quintessential sustainable development goal. Lancet 2013; 382:371-2.

4. Black RE, Alderman H, Bhutta ZA, Stuart Gillespie. Haddad L, Hortonet S, et al. Maternal and child nutrition: Building momentum for impact. Lancet 2013; 382:372-5.

5. UNICEF. The State of the World's Children 2014 in Numbers: Every child counts-Revealing disparities, advancing children's rights. New York: United Nations Children's Fund, 2014.

6. Park SE, Kim S, Ouma C, Mesfin Loha,Wierzba TF, Beck NS. Community management of acute malnutrition in the developing world. Pediatr Gastroenterol Hepatol Nutr 2012; 15:210-9.

7. Sameen I, Moorani K. Morbidity patterns of severely malnourished children At Tertiary Care Hospital. Pak Pediatr J 2014; 38(1): 3-8

8. National guidelines 2010, Nutrition Wing, Ministry of Health, Government of Pakistan, September 2010.

9. Hossain I,Dodd NS, Ahmed T, Miah GM, Jamil KM, Nahar $B$. Experience in managing severe malnutrition in a government tertiary treatment facility in Bangladesh Health Popul Nutr 2009; 27(1):72-9.
10. Ejaz MS, Ahmed IR, Huma Z. Clinical pattern of infections in malnourished children. Medical Channel 2010; 16(3): 352-6.

11. Abrha A, Abdissa A, Beyene G, Getahun G, Girma T. Bacteremia among severely malnourished children in Jimma University Hospital, Ethiopia. Ethiop J Health Sci. 2011; 21(3): 175-82.

12. Irena $\mathrm{AH}$, Mwambazi $\mathrm{M}$, Mulenga V. Diarrhea is a major killer of children with severe acute malnutrition admitted to inpatient setup in Lusaka, Zambia. Nutrition journel 2011; 10(1):110.

13. Latif $R$. Malnutrition in children in Low socioeconomic area of Karachi. Pak Ped J, 2007; 31(2): 53-57.

14. Ahmed S, Arif F, Akram DS. Psychomotor development of Malnourished versus healthy Pakistani children of up to 6 years of age. Pak Paed J 2008; 32(1): 23-28.

15. Guerrant RL, Oriá $R B$, Moore SR, Oriá MO, Lima AA. Malnutrition as an enteric infectious disease with long-term effects on child development. Nutr. Rev. 2008; 66: 487-505.

16. Mondal D, Haque R, Sack B, Kirkpatrick B, Petri W. Attribution of malnutrition to causespecific diarrheal illness: Evidence from a prospective study of preschool children in Mirpur, Dhaka, Bangladesh. Am JTrop Med Hyg 2009; 80: 824-26.

\begin{tabular}{|c|c|c|c|}
\hline \multicolumn{4}{|c|}{ AUTHORSHIP AND CONTRIBUTION DECLARATION } \\
\hline Sr. \# & Author(s) Full Name & Contribution to the paper & Author(s) Signature \\
\hline 1 & Waqas Ali & $\begin{array}{l}\text { Data collection, Data analysis, } \\
\text { Drafting. }\end{array}$ & $\cos \frac{\sin }{4}$ \\
\hline 2 & Asif Ali Khuhro & $\begin{array}{l}\text { Methodology, Literature } \\
\text { Review. }\end{array}$ & \\
\hline 3 & Sanam Bano Rajper & $\begin{array}{l}\text { Study concept, Supervision, } \\
\text { Proof reading. }\end{array}$ & 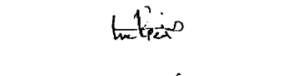 \\
\hline 4 & & Discussion, Data interpretation. & 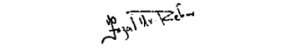 \\
\hline
\end{tabular}

